\title{
A Rare Case of Tamoxifen-Induced Thrombocytopenia
}

\author{
Anurag Pathak, Sushil Kiran Kunder, A Avinash, Navin Patil*, N Karthik Rao \\ Assistant Professor, Dept. of Pharmacology, Kasturba Medical College, Manipal 576104, India, Assistant Professor, Dept. of Medicine, Kasturba Medical \\ College, Manipal 576104, India.
}

\begin{tabular}{l} 
ARTICLE INFO \\
\hline Article history: \\
Received on: $16 / 10 / 2015$ \\
Revised on: $21 / 11 / 2015$ \\
Accepted on: $08 / 12 / 2015$ \\
Available online: $26 / 01 / 2016$
\end{tabular}

Key words: Low platelets, Anti-platelet antibodies, SERM, Breast cancer.

\begin{abstract}
Breast cancer is a commonly encountered cancer in women. Tamoxifen is a frequently used drug in breast cancer. It is a selective estrogen receptor modulator (SERM). It is used for both chemoprevention and for palliative care in these patients. Drug-induced thrombocytopenia is a common diagnosis these days. Moreover, this condition can be life threatening or even fatal. It can either be due to increased platelet destruction or reduced formation. Either way, early diagnosis followed by discontinuation of the offending agent is the key to successful management. Here, we present a rare case of tamoxifen-induced thrombocytopenia.
\end{abstract}

\section{INTRODUCTION}

The first oestrogen receptor (selective) modulator (SERM) to be introduced was tamoxifen. It acts as a partial agonist/antagonist at the oestradiol receptor. Even though the mechanism is studied extensively, it has not been understood completely till date. In women with high risk of developing carcinoma of breast, it is used for chemoprophylaxis. It is also used extensively in post-menopausal women for palliative management. It also reduces the chances of development of breast cancer in the contralateral breast. Nausea, vomiting and hot flushes are the usually encountered adverse effects of this SERM (Chrousos, 2015). Thrombocytopenia secondary to drug use is a common scenario we come across these days. In these cases, early diagnosis is vital before clinically significant manifestations (like bleeding) set in. Drug-induced thrombocytopenia is either immune mediated (increased destruction of platelets) or due to suppression of bone marrow (reduced formation of platelets) (Visentin and Liu, 2007).

\footnotetext{
* Corresponding Author

Dr. Navin Patil, Assistant Professor, Dept. of Pharmacology, Kasturba Medical College, Manipal 576104, India.Email: navin903@gmail.com
}

In case of thrombocytopenia, which is acute in onset, drugs as an aetiological factor should always be kept in mind. Even though heparin, carbamazepine and sulphonamidesare usually reported to cause such reactions, it should be kept in mind that several other drugs, including herbals and non-prescription drugs have also been implicated (Aster and Bougie, 2007).

\section{CASE REPORT}

A 44-year-old lady, a case of carcinoma of breast (diagnosed two years ago), post modified radical mastoidectomy and adjuvant chemo-radiotherapy, was started on oral tamoxifen 20mg dailyin December 2012. Three years later, she presented with two months history of multiple skin rashes (ecchymotic patches) and oral bleeding. Systemic examination was normal. Her platelet count on admission was 77,000/microlitre, haemoglobin level was $12.3 \mathrm{~g} / \mathrm{dl}$, total leucocyte count was 5400 cells/cu. mm., and ESR was elevated (54 mm/hr). Anti-nuclear antibody and antiphospholipids antibody were positive. On local examination of the right breast, hyperpigmentation was seen. A diagnosis of tamoxifen-induced thrombocytopenia was made. She was started on systemic steroids (prednisolone). The offending drug (tamoxifen) was continued. The platelet count on day 2 was 81,000 and 78,000 a day later. The 
patient was discharged two days later, with a platelet count of 96,000 . She was discharged on oral tamoxifen, oral pantoprazole and oral prednisolone. She was advised to come back for a followup after a fortnight.

On her subsequent visit, her platelet count was 1,34,000. She was sent back on the same medication. Two months later, on her next visit, her platelet counts were 1,06,000. Dose of prednisolone was halved from $20 \mathrm{mg}$ daily to $10 \mathrm{mg}$ daily.

\section{DISCUSSION}

On reviewing the available literature, we came across two cases of thrombocytopenia caused by tamoxifen (Chrousos, 2015; Yao et al., 1999). This reaction is postulated to be due to a possible immunological mechanism where drug dependent antibodies target against the platelets are produced (Nasiroglu et al., 2007). In presence of the drug, these antibodies bind to the platelet surface glycoproteins and destroy them (Visentin and Liu, 2007).

There are reports stating that tamoxifen use is also associated with reduced platelet function due to reduced peroxynitrite and increased platelet nitric oxide, there by resulting in an increase in the bleeding time (Scognamiglio et al., 2007). So, tamoxifen therapy can affect both the quality and the quantity of platelets in susceptible individuals (Nayak and Schmaier, 2012).

\section{CONCLUSION}

Even though thrombocytopenia secondary to tamoxifen use in breast carcinoma patients is a rare entity, it cannot be taken lightly as it can be fatal due to uncontrolled and excessive bleeding manifestations. Therefore, its ability to cause low platelet count should always be kept in mind when this drug is prescribed to patients, hence making monitoring of the platelet counts mandatory from time to time.

\section{REFERENCES}

Aster RH, Bougie DW. Drug induced immune thrombocytopenia. New England Journal of Medicine, 2007; 357:580-587.

Chrousos GP. 2015. The gonadal hormones and inhibitors. In: Katzung BG, Trevor AJ. Basic and clinical pharmacology. McGraw Hill. 1047.

Nasiroglu N, Pamukçuoğlu M, Abali H, Oksuzoglu B, Uner A, Zengin N. Tamoxifen induced-thrombocytopenia: it does occur. Medical Oncology, 2007; 24(4):453-4.

Nayak L, Schmaier AH. A Platelet Acquired Storage Pool Disorder Associated with Tamoxifen Therapy. Case Reports in Hematology, 2012; 2012: 948351

Scognamiglio F, Corso C, Madeo D, Castaman G, Visco $\mathrm{C}$, Borghero C. Flow cytometry in the diagnosis of drug induced thrombocytopenia: two illustrative cases. American Journal of Hematology, 2007; 83: 326-9.

Visentin GP, Liu CY. Drug Induced Thrombocytopenia. Hematology/Oncology Clinics of North America, 2007;21(4):685.

Yao JC, Thomakos N, McLaughlin P, Buchholz TA, Kudelka AP. Tamoxifen induced thrombocytopenia. American Journal of Clinical Oncology, 1999; 22(5):529-32.

\section{How to cite this article:}

Pathak A, Kunder SK, Avinash A, Patil N, Rao NK. A Rare Case of Tamoxifen-Induced Thrombocytopenia. J App Pharm Sci, 2016; 6 (01): 156-157. 